\title{
Short-term memory and presentation rates with young children
}

DUNCAN N. HANSEN

STANFORD UNIVERSITY

\begin{abstract}
Abstraet
Two groups of children, a 5-year-old and a 10-yearold group, were run in a short-term memory experiment designed to determine the effect of presentation rate on retention. The results indicate that longer presentation intervals improve recall of recently presented items while shorter presentation intervals improve recall of early items.
\end{abstract}

\section{Problem}

In short-term memory (STM) studies it is a common finding that the recall of an item is a decreasing function of the number of interpolated items between presentation and testing of the specific item (Posner, 1963). Moreover, it has been found that the rate of presentation and its consequent inter-item interval has little or no effect on the forgetting of an item (Murdock, 1961; Waugh \& Norman, 1965). This study was designed to test whether these findings could be generalized to include a wider age range, specifically young children. The STM procedure was designed so that the interpolated material was from the same class of relevant stimuli. The variation of the presentation rates ( 1 and 3 sec.) offered differential opportunities for rehearsal of the stimuli. The overall experimental procedure was nearly equivalent to a previously employed one that resulted in the collection of meaningful STM data from your children (Atkinson, Hansen, \& Bernbach, 1964).

\section{Method}

Forty children served as S's in the experiment. Group I consisted of 10-year olds and Group II of 5-year olds who were attending a child care center. Each group consisted of 10 boys and 10 girls. For Group I, the Ss were $9-3 / 4$ to $10-1 / 2$ years old (mean age $=10.09$ ); for Group II the age ranges were $4-1 / 2$ to $5-1 / 2$ years (mean age $=5.13$ ).

Materials to be remembered consisted of eight animal cards, that is, playing cards containing pictures of familiar animals. On each trial the eight cards were randomly presented, being shown one at a time to $\mathrm{S}$. Two rates of presentation were employed, a 1-sec. and a 3-sec. rate. After each card was shown to $\mathrm{S}$, it was placed face down on the table so that after all eight cards were presented they formed a horizontal row in front of S. After the last card was laid down, a second array of eight randomly arranged but identical face-up cards was presented to the S. E pointed to one of the face-down cards and asked $S$ to point to a card on the face-up array that "was the same." $S$ responded by pointing to one of the face-up cards on the response array. After $\mathrm{S}$ made his response, the face-down card

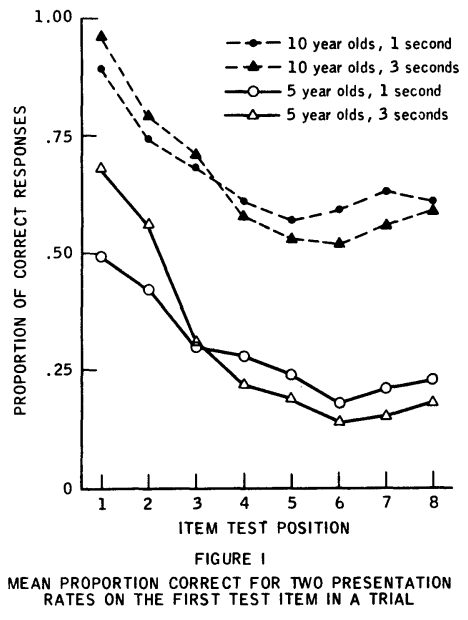

was exposed and placed on the face-up card response array. S was then quizzed about the card adjacent to the initial test item. The sequence of E's inquiry pointing and S's response pointing was identical for both the first and second test items. The second test item was systematically varied as to prior and subsequent position in the lay-down array.

The assignment of pictures to serial position was randomly determined for each trial. The eight different face-up card response arrays that were employed were constructed so that each animal card appeared in the two end positions at least once. The intermediate cards were randomly assigned. Each $\mathrm{S}$ was run for 32 trials; a trial consisted of the presentation and testing of both the first and second test items. For the first test item, each of the eight card positions were tested four times in a session, two data points from each presentation rate. For the second test item on a trial, the distribution of adjacent test positions was uniformly distributed except for positions 1,2, 7, and 8. These were unequal since the only adjacent cards to 1 and 8 were the positions 2 and 7. The response of the child on each test item was recorded. For the second test item, the prior first test card had been turned over so that the child was able to see whether he was correct or incorrect; consequently, the guess probability on the second item was 1 out of 7 .

\section{Results}

Figure 1 presents the proportion of correct responses for the first test item at each position for the two groups of Ss. Position 1 corresponds to the most re- 
cently displayed card and position 8 to the card first shown on a trial. As expected, the proportion of correct responses at a given position is a decreasing function of the number of items intervening between the presentation of the item and its test for recall. There was evidence for a slight primacy effect. There was a significant difference between the two age groups $(p<.001)$. The most interesting finding was the systematic cross-over of the curves for the two presentation rates; this finding held for both age groups. In the analysis of variance, a significant interaction between presentation rate and amount of interpolated material was found $(p<.01)$.

Figure 2 presents the proportion correct by position for the second test item in a given trial. Forgetting again was an increasing function of the amount of the interpolated material except for a slight primacy effect. The curves for the second test items were systematically superior to those of the first test items, even when corrected for guessing ( $P$ (recall $)=P$ (correct) $-\mathrm{g} /(1-\mathrm{g})$ where $\mathrm{g}=1 / \mathrm{NO}$. of alternatives). Two additional findings were (1) the superiority of the $3-\mathrm{sec}$. over the $1-\mathrm{sec}$. presentation rate and (2) the absence of any cross-over between the two presentation curves. Again there was a significant difference between the two age groups.

\section{Discussion}

As in STM studies with adults, the recall of items is a decreasing function of the number of interpolated items between presentation and testing. This held for first and second test items on a trial. There was a slight primacy effect that would qualify this generalization.

More importantly, the results indicated that the rate of presentation ( 1 or 3 sec.) did influence the recall function in two ways: (1) the longer presentation intervals provided superior recall in the recency positions (No. 1, 2, and 3) for the first test item on a trial and (2) the 3 -sec. presentation rate was consistently superior for second test items.

A theoretical formulation of separate short-term and long-term memory (LTM) processes (Atkinson \& Crothers, 1964; Waugh \& Norman, 1964) provides a parsimonious interpretation of the presentation rate effects. The observed recall curves are hypothesized to be the resulting combination of a STM negative accelerating exponential decay function and LTM serial learning curve. The longer presentation interval allows $\mathrm{S}$ to rehearse more of the recent items in the limited

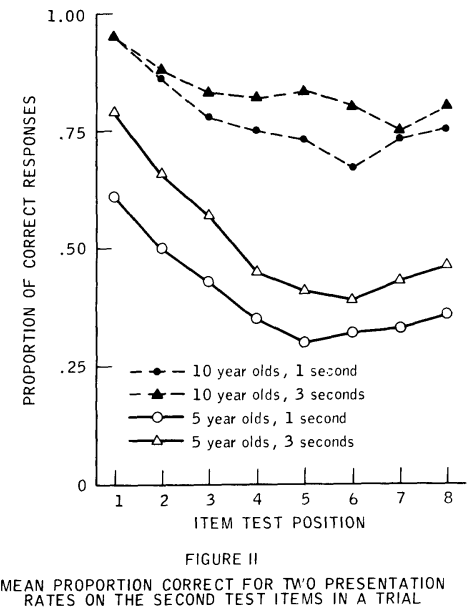

capacity STM system (Mackworth, 1959) and consequently effectively remove the earlier items by interference effects. Thus the 3-sec.presentation rate items would be superior to the $1-$ sec. presentation rate items in the recency positions (No.1,2, and 3). The 1-sec. presentation rate items would be recalled most readily in the earlier positions because there is less likelihood of the interference effects removing them. The primacy effect in the early positions would be attributed to the hypothesized LTM serial learning curve.

If one assumes that rehearsal increases the availability of the information in the LTM system, then the superiority of the 3-sec. presentation rate items in the second test item curves is explained. The overall superiority of the second test item performance can be attributed to the cueing or prompting value of knowing the first test item. Thus the contributions from the LTM system are maximized if a cueing stimuli is available for aid in the search of this more permanent information system.

\section{References}

ATKINSON, R. C., HANSEN, D. N., \& BERNBACH, H. A. Short-term memory with young children. Psychon. Sci., 1964, 1, 255-256.

ATKINSON, R. C., \& CROTHERS, E. J. A comparison of pairedassociate learning models having different acquisition and retention axioms. J. math. Psychol., 1964, 1, 285-315.

MACKWORTH, J.F. Paced memorization in a continuous task. J. exp. Psychol., 1959, 58, 206-212.

MURDOCK, B. B. The retention of individual items.J. exp. Psychol., $1961,62,618-625$.

POSNER, M. I. Immediate memory in sequential tasks. Psychol. Bull., 1963, 60, 333-349.

WAUGH, N. C., \& NORMAN, D。A. Primary memory. Psychol. Rev., 1965, 72, 89-104. 